\title{
Effect of guar gum on body-weight, hunger ratings and metabolism in obese subjects
}

\author{
BY MARCIN KROTKIEWSKI \\ Department of Rehabilitation Medicine, Sahlgren's Hospital, University of Göteborg, \\ Göteborg, Sweden
}

\section{(Received 29 September 1983 - Accepted 29 February 1984)}

\begin{abstract}
1. The effect of a palatable granulated guar-gum preparation (10 $\mathrm{g}$ twice daily) was studied in obese subjects. The acute effect of a single dose of guar gum to reduce the peak postprandial whole blood glucose levels (about $10 \%$ ) was verified. Following long-term treatment, a further reduction was seen in the obese subjects with the highest postprandial glucose levels. Since the postprandial plasma insulin levels were essentially unchanged, this finding suggested an increased responsiveness to insulin. Total serum cholesterol levels were significantly reduced following long-term treatment but serum $\alpha$-cholesterol levels, representing the high-density-lipoprotein fraction, was unchanged.

2. Body-weight was significantly reduced during guar-gum treatment even though the patients were asked to maintain their normal dietary habits. Daily hunger ratings recorded for up to 10 weeks showed that guar gum reduced hunger significantly better than commercially available bran taken in the same way.

3. Thus, guar gum seemed to influence carbohydrate and lipid metabolism in a beneficial way in obese subjects. The reduction in hunger would offer an additional benefit to these patients.
\end{abstract}

The various effects of dietary fibre have attracted great interest during recent years. It has become increasingly clear that the metabolic effects of different types of fibre may vary. Gel-forming fibres, such as guar gum, have been shown to reduce postprandial glucose and insulin levels in healthy volunteers (Jenkins et al. 1977, 1978; Smith \& Holm, 1982) and in diabetic subjects (Jenkins et al. 1976; Morgan et al. 1979; Smith \& Holm, 1982). Likely reasons for this are a reduced rate of carbohydrate absorption due to a slower gastric emptying rate (Holt et al. 1979; Wilmshurst \& Crawley, 1980) as well as a reduced secretion of insulin secretagogues like gastric inhibitory polypeptide (GIP; Morgan et al. 1979).

Reduction in low-density-lipoprotein (LDL)-cholesterol, possibly due to increased faecal loss, while high-density-lipoprotein (HDL)-cholesterol levels were unchanged, have also been reported following guar-gum treatment (Jenkins et al. 1979; Smith \& Holm, 1982).

Obese subjects are at an increased risk of developing diabetes and they frequently have abnormal lipid metabolism. Thus, due to its effects on metabolism, guar gum may prove a useful adjunct to conventional weight-reducing therapy in obese individuals. Apart from the effects on carbohydrate and lipid metabolism, guar gum may offer a particular benefit to obese patients since a slower rate of gastric emptying may increase satiety. In the present study obese subjects were treated with a specially prepared, palatable granular guar gum over a prolonged period of time. The objectives were to evaluate the effects of guar gum on postprandial blood glucose levels as well as on fasting lipid levels in obesity. In addition, the effect of guar gum on body-weight and hunger was also considered. Since no placebo with the same physico-chemical characteristics as guar gum is known the results were compared with those obtained under the same conditions with a non-gelling fibre like wheat bran.

A single dose of guar gum has been shown to reduce postprandial glucose levels in normal subjects (Jenkins et al. 1977). However, in a later study (Smith \& Holm, 1982) it was found that long-term treatment with guar gum in diabetic subjects produced additional effects, since fasting glucose levels were also reduced and the postprandial glucose levels were further attenuated. 
Table 1. Clinical characteristics of the patients participating in the metabolic studies

\begin{tabular}{ccccc}
\hline \hline $\begin{array}{c}\text { Patient } \\
\text { no. }\end{array}$ & $\begin{array}{c}\text { Age } \\
\text { (years) }\end{array}$ & $\begin{array}{c}\text { Relative } \\
\text { body-wt* }(\%)\end{array}$ & $\begin{array}{c}\text { Body fat } \\
(\mathrm{kg})\end{array}$ & $\begin{array}{c}\text { Body-wt } \\
(\mathrm{kg})\end{array}$ \\
\hline 1 & 31 & 127 & $30 \cdot 0$ & $73 \cdot 5$ \\
2 & 53 & 154 & $46 \cdot 8$ & $110 \cdot 0$ \\
3 & 51 & 162 & $50 \cdot 4$ & $95 \cdot 3$ \\
4 & 57 & 169 & $46 \cdot 0$ & $90 \cdot 5$ \\
5 & 34 & 195 & $45 \cdot 8$ & $72 \cdot 5$ \\
6 & 55 & 159 & $43 \cdot 0$ & $93 \cdot 5$ \\
7 & 31 & 167 & $41 \cdot 6$ & $99 \cdot 5$ \\
8 & 47 & 195 & $63 \cdot 6$ & $94 \cdot 4$ \\
9 & 30 & 187 & $48 \cdot 1$ & $103 \cdot 0$ \\
\hline
\end{tabular}

* Calculated from ideal body-weight according to Metropolitan Life Insurance Co. (1959) weight standards.

The present study was designed to assess both these effects in obese subjects. Thus the acute effect of guar gum on postprandial glucose levels in connection with a standardized test meal was studied. In order to evaluate the long-term effects the patients were treated with guar gum for 8 weeks. The same test meal was again given to study whether fasting levels or postprandial peaks were further attenuated.

\section{MATERIAL AND METHODS}

\section{Expt 1. Metabolic studies}

Patients. Nine obese female subjects were studied. They were recruited from patients just referred to the obesity out-patient clinic for weight reduction. Their ages ranged from 30 to 57 years and their body-weight from 72.5 to $110.0 \mathrm{~kg}$. Further details are shown in Table 1. Four patients (nos. 3-5, 8) were receiving diuretics and beta-blocking drugs for treatment of peripheral oedema and hypertension. Their medication remained unchanged during the study. The patients were told that the main aim of the study was to evaluate the lipid-lowering effect of guar gum.

Experimental procedures. Total body potassium content was measured in all patients in a whole-body counter (Nuclear Enterprises, Edinburgh) detecting naturally occurring ${ }^{40} \mathrm{~K}$; correction was made for the shielding effect of body fat by ${ }^{42} \mathrm{~K}$ administration (Sköldborn \& Arvidsson, 1972). Lean body mass (LBM) was calculated on the assumption that the $\mathrm{K}$ content is $68.1 \mathrm{mmol} / \mathrm{kg}$ (Forbes et al. 1961). Body fat was then estimated by subtracting LBM from the body-weight.

Venous blood samples were drawn after an overnight fast for the determination of serum triglycerides (Wahlefeld, 1974), serum cholesterol (Borner \& Klose, 1977), serum $\alpha$-cholesterol (Wiklund et al. 1980) and whole blood glucose (glucose oxidase (EC 1 . 1 .3 .4); KABI, Stockholm, Sweden). The patients were then given a standardized meal (TV-dinner) of $350 \mathrm{ml}$ low-fat milk supplying $243 \mathrm{~kJ}$ (577 kcal) and $41.5 \mathrm{~g}$ carbohydrate, $27 \mathrm{~g}$ fat and $15 \mathrm{~g}$ protein according to a previously published regimen (Smith \& Holm, 1982). Granulated guar gum (10 g, Lej Guar; Lejus Medical, Gothenburg, Sweden) was stirred in $150 \mathrm{ml}$ water and given immediately before the test meal. The time required to consume the test meal was measured and was less than $10 \mathrm{~min}$ in all patients.

Blood samples were drawn at the time-intervals indicated (Figs. 1-3) after the completion of the meal for the determinations of blood glucose and plasma insulin (Phadebas; Pharmacia, Uppsala, Sweden).

Acute study. The acute effect of a single dose of guar gum on the postprandial glucose 
levels was evaluated in five of the obese patients. In that study the standardized meal was given to the patients either with or without $10 \mathrm{~g}$ granulated guar gum stirred in $150 \mathrm{ml}$ water. The order of treatment was randomized and the studies performed with at least a 1 week interval. During this period no other guar gum was given.

Long-term study. Following the initial test meal the patients were given the granulated guar gum to take at home ( $10 \mathrm{~g}$ twice daily) stirred in water immediately before the two main meals (usually lunch and dinner). They were asked explicitly not to alter their normal diet or energy intake during the trial period. Diet records $(4 \mathrm{~d})$ before and during guar-gum treatment suggested that no major qualitative difference in dietary habits occurred although the patients did report on differences in hunger as discussed in detail later (p. 102). After 8 weeks' treatment, body-composition studies were again performed as described previously. Fasting venous blood samples were drawn for the determination of serum lipids and blood glucose. The standardized meal was again given and the time required to consume the meal was kept similar to that required on the first occasion Granulated guar gum (10 g) was again given in $150 \mathrm{ml}$ water immediately before the meal. Blood samples were drawn at the indicated time-intervals for the determination of blood glucose and plasma insulin.

It should be emphasized that the design of the long-term study was aimed at studying any additional effect achieved by long-term guar-gum treatment on blood glucose levels compared with the acute-study effects, since guar gum was given before each test meal.

\section{Expt 2. Hunger ratings}

Since the obese patients in Expt 1 lost weight (see Table 3) and several patients spontaneously reported an increased satiety during guar-gum treatment a second experiment was carried out.

Patients. Twenty-one obese patients, two males and nineteen females, were recruited from the obesity out-patient clinic. They had all been referred for weight reduction. Their age ranged from 22 to 61 years, body-weight from 71 to $155 \mathrm{~kg}$, body fat from 26.5 to $74.3 \mathrm{~kg}$ and relative over-weight (Metropolitan Life Insurance Co. 1959) from 150 to $195 \%$.

Experimental procedures. Body-composition studies were performed as described previously. The patients were then asked to supply daily ratings of their hunger before breakfast, lunch, dinner and possible evening snack according to a scale graded from 1 to 9. Rating 1 implied 'no desire to eat' and rating 9 'unbearable hunger - must eat immediately' (see Table 2). The patients were asked to maintain their normal dietary habits throughout the study.

The patients were given either $10 \mathrm{~g}$ wheat bran (of $0.2-1.5 \mathrm{~mm}$ size fraction and with a neutral-detergent fibre content of $400 \mathrm{~g} / \mathrm{kg}$ ) twice daily stirred in $150 \mathrm{ml}$ water before the two main meals for 1 week, or $10 \mathrm{~g}$ guar gum twice daily administered in water in the same way. Treatment was switched each week to allow an adequate comparison of their daily hunger sensations with the two treatments. This design does not eliminate possible carry-over effects of the different treatments, but the repeated observations performed would minimize this potential error.

On average, hunger ratings were noted by the patients for 6.6 weeks $(3.3$ weeks on each treatment). Seven patients continued with the ratings for 10 weeks ( 5 weeks on each treatment).

Body-weight was measured each week before treatment was switched and the results of the hunger ratings during the week collected.

Statistical methods. Conventional statistical methods were used. Significance of difference was estimated with Student's $t$ test for paired values, since normally distributed variables were measured. $P<0.05$ was considered statistically significant and $P<0.1$ as probably significant, but such significance levels should be interpreted with some caution. 


\section{Table 2. Definitions of the hunger ratings}
1. No desire to eat
2. Can eat but also refrain
3. Can eat but only little
4. Want to eat but can wait
5. Yearning for something special
6. Feel hungry but only for a certain food
7. Feel hungry and yearn for food - want to eat shortly
8. Feel very hungry
9. Unbearable hunger - must eat immediately

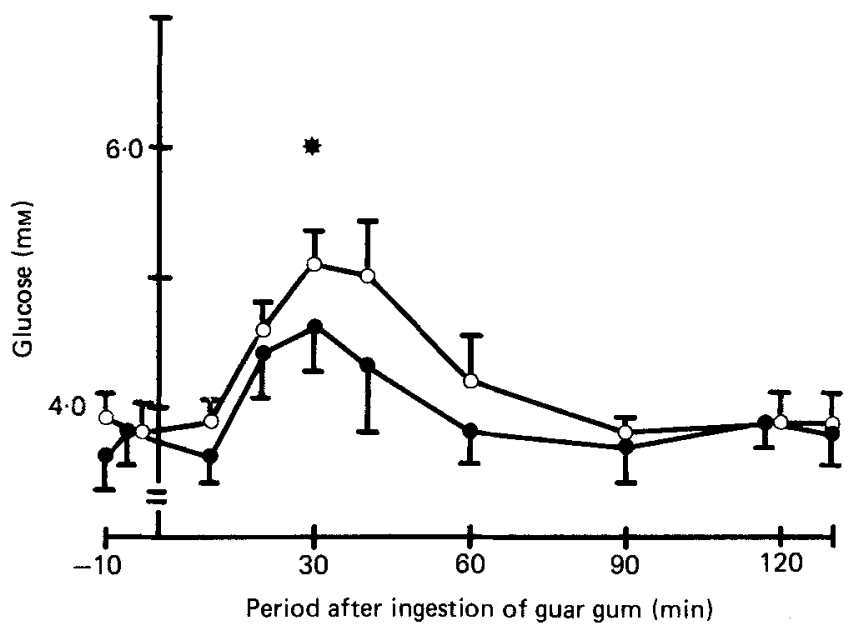

Fig. 1. Blood glucose levels (mM) in five obese subjects following a standardized meal with (O) and without $(O)$ ingestion of $10 \mathrm{~g}$ granulated guar gum taken immediately before the test meal. Points are mean values, with their standard errors represented by vertical bars. ${ }^{*} P<0.05$.

\section{RESULTS}

Metabolic investigations

Acute study. The acute effect of $10 \mathrm{~g}$ guar gum on postprandial blood glucose levels following a standardized meal in five obese patients is shown in Fig. 1. The peak postprandial glucose levels (at $30 \mathrm{~min}$ ) were reduced by about $10 \%$ by guar-gum treatment (5.1 v. $4.6 \mathrm{mmol} / 1 ; P<0.05)$.

Long-term study. As shown in Table 3, there was a significant decrease in body-weight and body fat in the obese subjects after 8 weeks' treatment with the granulated guar gum. The total cholesterol levels were significantly reduced but the $\alpha$-cholesterol as well as the triglyceride levels remained unchanged.

The mean postprandial peak glucose level, reached at $30 \mathrm{~min}$, was significantly reduced following long-term guar-gum treatment (Fig. 2a). This reduction, however, was due to the changes seen in two patients whose initial postprandial glucose levels were higher than the mean values $( \pm 2 \mathrm{SD}$ ) for the whole group $(8.0$ and $8.8 \mathrm{mmol} / 1$ respectively). The results for these two patients are shown in Fig. $2 b$. Following guar-gum treatment the glucose levels were reduced by 19 and $26 \%$ in these two patients and were then well within the mean values $( \pm 2 \mathrm{SD})$ of the pretreatment values (Fig. $2 a$ ). If the results shown in Fig. $2 a$ were recalculated with the omission of these two patients, postprandial blood glucose levels were 
Table 3. Body-weight, body fat, serum total cholesterol, $\alpha$-cholesterol and triglycerides before and after 8 weeks of treatment with guar gum

(Mean values with their standard errors for nine obese subjects)

\begin{tabular}{|c|c|c|c|c|c|}
\hline & \multicolumn{2}{|c|}{$\begin{array}{c}\text { Before } \\
\text { treatment }\end{array}$} & \multicolumn{2}{|c|}{$\begin{array}{c}\text { After guar } \\
\text { gum treatment }\end{array}$} & \multirow{2}{*}{$\begin{array}{c}\text { Statistical } \\
\text { significance of } \\
\text { difference: } P\end{array}$} \\
\hline & Mean & $\mathbf{S E}$ & Mean & SE & \\
\hline Body-wt (kg) & $95 \cdot 6$ & $3 \cdot 1$ & $91 \cdot 3$ & $2 \cdot 1$ & $<0.01$ \\
\hline Body fat $(\mathrm{kg})$ & $46 \cdot 1$ & 2.9 & $43 \cdot 6$ & $2 \cdot 8$ & $<0.01$ \\
\hline Cholesterol (mM) & $6 \cdot 2$ & $0 \cdot 3$ & 5.7 & 0.4 & $<0.05$ \\
\hline$\alpha$-Cholesterol (mM) & $1 \cdot 3$ & $0 \cdot 1$ & $1 \cdot 2$ & 0.1 & NS \\
\hline Triglycerides (mM) & 1.8 & 0.2 & $1 \cdot 7$ & 0.2 & NS \\
\hline
\end{tabular}

NS, not significant.
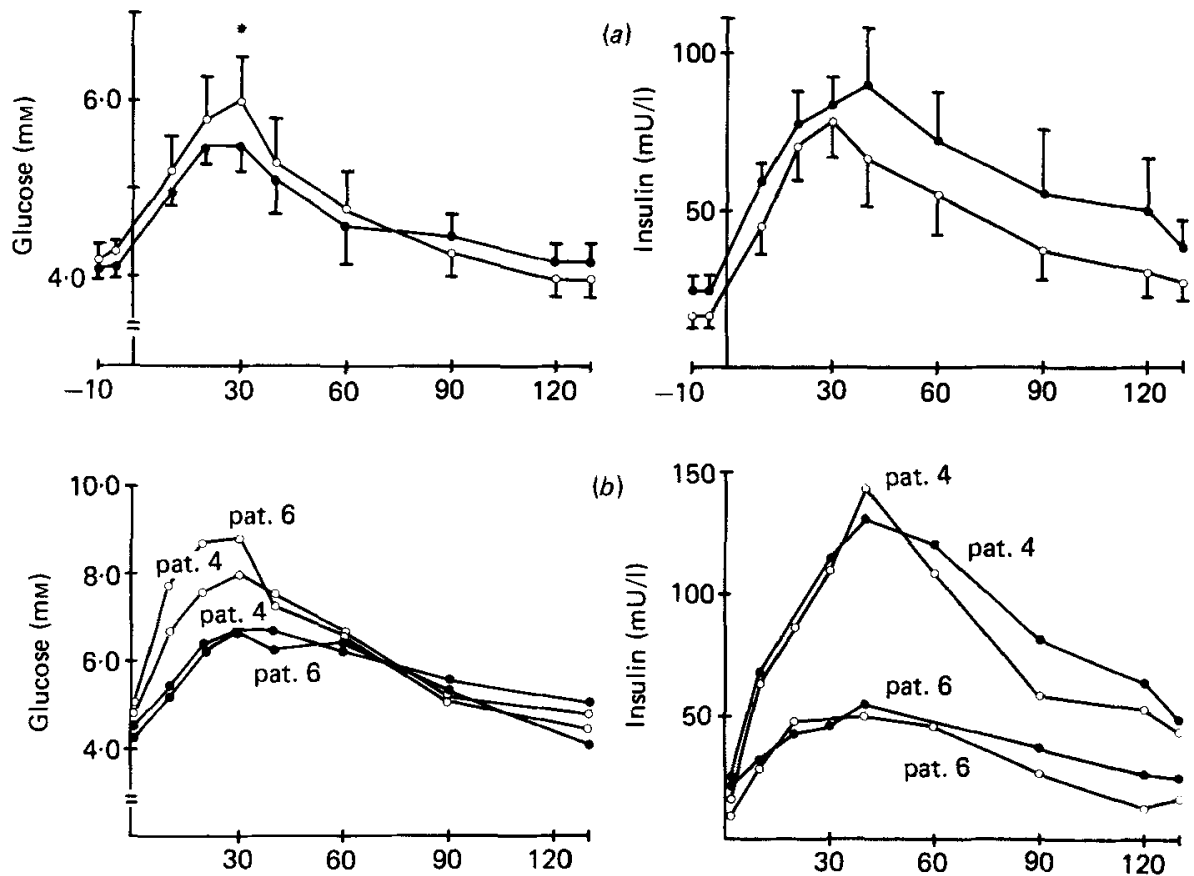

Period after ingestion of guar gum ( $\mathrm{min}$ )

Fig. 2. (a) Blood glucose (mM) and insulin levels ( $\mathrm{mU} / \mathrm{l})$ following a standardized meal in nine obese subjects. Granulated guar gum $(10 \mathrm{~g})$ was given immediately before the meal. $(O)$, Before treatment; (O), after 8 weeks of treatment with guar gum, $10 \mathrm{~g}$ twice daily. Points are mean values, with their standard errors represented by vertical bars. ${ }^{*} P<0 \cdot 05$. (b) Blood glucose (mM) and insulin levels (mU/1) before $(O)$ and after $(O) 8$ weeks of guar gum treatment in the two subjects (pat. 4 and pat. 6) whose pretreatment peak postprandial levels were outside the mean \pm 2 SD of the whole group.

not significantly different before and after the 8 -week treatment period. Similar peak values were also reached $(5 \cdot 3 \mathrm{mmol} / \mathrm{l})$.

The fasting serum insulin levels were not significantly changed by guar-gum treatment. The mean postprandial increase in serum insulin levels (Fig. 2a) was also similar before and after long-term treatment (peak increases 62 and $66 \mathrm{mU} / 1$ respectively). The insulin 


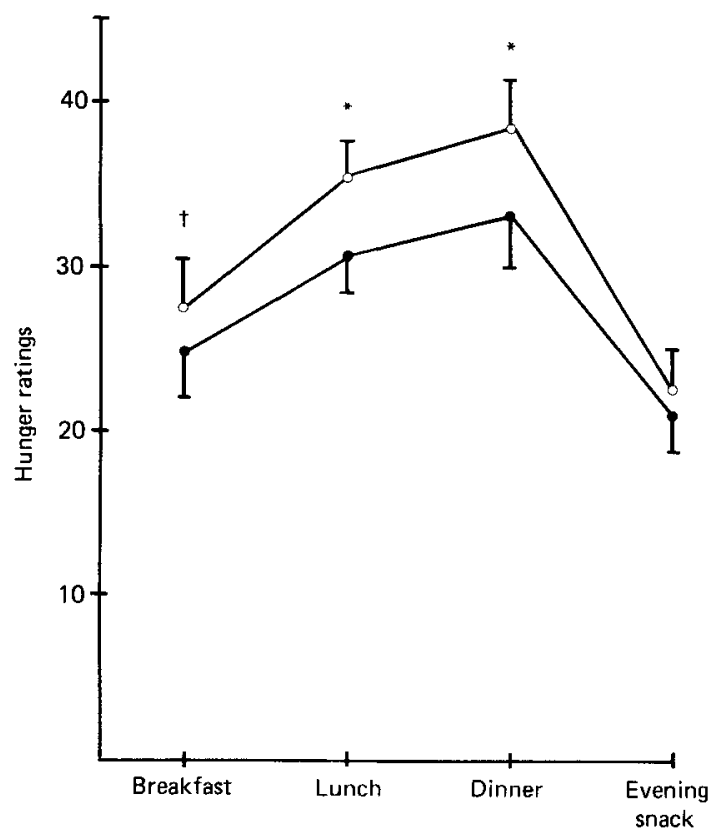

Fig. 3. Hunger ratings (see Table 2 ) in twenty-one obese subjects given $10 \mathrm{~g}$ guar gum twice daily (O) or wheat bran $(\mathrm{O})$ at the same time as their main meals. Treatment was alternated each week. Points are the mean values with their standard errors for an average observation period of 3.3 weeks and are expressed as the sum of weekly hunger ratings. $\uparrow P<0 \cdot 1,{ }^{*} P<0 \cdot 05$.

levels were similar in the two obese patients who showed the greatest decrease in postprandial glucose levels (Fig. $2 b$ ).

\section{Hunger ratings}

The mean accumulated weekly hunger ratings reported by the twenty-one patients on guar-gum and wheat-bran treatment for an average period of 3.3 weeks each are shown in Fig. 3. Clearly, lower weekly hunger ratings were reported during guar-gum treatment than during bran treatment. This was particularly pronounced during lunch and dinner when most patients took their respective treatments.

Seven patients were able to record their hunger ratings for 5 weeks each on both guar-gum and bran treatment. A similar reduction in hunger ratings was maintained (Table 4).

Body-weight was significantly reduced during the study (Table 5) even though the patients were specifically asked not to alter their dietary habits. The mean weekly reduction in body-weight tended to be greater during guar-gum treatment than during wheat-bran treatment (Table 5).

Side effects of treatment. The reported side effects were those to be expected from an increased fibre intake, i.e. increased flatulence and loose stools. The side effects were usually transient and improved after 2-3 weeks or following a temporary reduction in dose.

\section{DISCUSSION}

The present study was designed to elucidate the long-term metabolic effects of guar-gum treatment in obesity. Before the long-term therapy was started, guar gum was given with a test meal to patients. In this way, the acute effect of guar gum of lowering the postprandial 
Table 4. Body-weights and average hunger ratings (sum per week) in obese patients treated with guar gum or wheat bran (10 g twice daily) for 10 weeks

\begin{tabular}{|c|c|c|c|c|c|c|c|c|c|c|}
\hline \multirow{3}{*}{$\begin{array}{c}\text { Patient } \\
\text { no. }\end{array}$} & \multirow{2}{*}{\multicolumn{2}{|c|}{ Body-wt (kg) }} & \multicolumn{8}{|c|}{ Hunger ratings } \\
\hline & & & \multicolumn{4}{|c|}{ Guar gum } & \multicolumn{4}{|c|}{ Bran } \\
\hline & Initial & $\begin{array}{c}\text { Atter } \\
10 \text { weeks }\end{array}$ & B & $\mathrm{L}$ & $\mathrm{D}$ & $\mathrm{E}$ & B & $\mathrm{L}$ & $\mathrm{D}$ & $\mathrm{E}$ \\
\hline 1 & $73 \cdot 5$ & $66 \cdot 0$ & 44 & 33 & 40 & 15 & 55 & 51 & 52 & 16 \\
\hline 2 & $110 \cdot 0$ & $96 \cdot 1$ & 18 & 23 & 28 & 28 & 24 & 27 & 31 & 28 \\
\hline 3 & $95 \cdot 3$ & $90 \cdot 6$ & 45 & 40 & 43 & 37 & 44 & 37 & 46 & 36 \\
\hline 4 & $90 \cdot 5$ & $88 \cdot 4$ & 20 & 43 & 39 & 1 & 25 & 51 & 53 & 1 \\
\hline 5 & $72 \cdot 5$ & $67 \cdot 5$ & 16 & 24 & 35 & 27 & 19 & 28 & 33 & 32 \\
\hline 6 & $93 \cdot 5$ & $89 \cdot 0$ & 11 & 38 & 38 & 19 & 13 & 36 & 40 & 17 \\
\hline 7 & $99 \cdot 5$ & $88 \cdot 0$ & 21 & 21 & 21 & 7 & 21 & 21 & 21 & 7 \\
\hline Mean & $90 \cdot 7$ & $83 \cdot 7^{*}$ & 25 & 32 & 35 & 19 & $29^{*}$ & 36 & $39 \dagger$ & 18 \\
\hline
\end{tabular}

B, L, D, E, hunger ratings before breakfast, lunch, dinner and evening snack respectively. For details of hunger ratings, see Table 2.

Mean values were significantly different from the initial value (body-weight) or from the corresponding value for guar-gum treatment: $\dagger P<0 \cdot 1,{ }^{*} P<0 \cdot 05$.

Table 5. Body-weight in obese subjects before and after weekly alternating treatment with guar gum or wheat bran (10 g twice daily) for 10 weeks

(Mean values with their standard errors)

Nine patients were treated for 1 week with guar gum or wheat bran in an alternating regimen before the main meals for a total period of 10 weeks (i.e. 5 weeks on each agent). Body-weight was recorded after each week

\begin{tabular}{clll}
\hline & Mean & SE \\
Body-wt (kg): & & \\
$\quad$ Initial & $92 \cdot 5$ & $4 \cdot 2$ \\
$\quad$ After 10 weeks & $85 \cdot 4^{* *}$ & $3 \cdot 7$ \\
Body-wt reduction & & \\
(kg/week) & & \\
Guar gum & 0.94 & $0 \cdot 2$ \\
Wheat bran & $0.64 \dagger$ & $0 \cdot 1$ \\
\hline
\end{tabular}

$$
\dagger P<0 \cdot 1, * * P<0 \cdot 01
$$

glucose levels was maintained at each test meal and potential effects above and beyond this, such as long-term improvement in carbohydrate tolerance, could be studied. It is clear that a single dose of guar gum reduces postprandial, but not fasting, glucose levels when given in immediate conjunction with meals (Jenkins et al. 1977, 1978; Smith \& Holm, 1982). This was also found in the five obese patients where this acute effect was specifically studied. The peak postprandial glucose levels were significantly reduced by about $10 \%$.

Interestingly, however, long-term guar-gum treatment further reduced the postprandial glucose levels in the two obese subjects who exhibited a reduced glucose tolerance as compared with the group as a whole (Fig. $2 b$ ). This effect was reached in spite of unchanged insulin levels suggesting a reduction in peripheral insulin resistance and an improvement in carbohydrate tolerance.

It is unlikely that the slight weight reduction in these subjects (from 95.6 to $91.3 \mathrm{~kg}$ ) could be responsible for the marked reduction in glucose levels. In addition, comparable results 
have been obtained in diabetics and subjects with reduced glucose tolerance in spite of unchanged body-weight (Jenkins et al. 1976; Smith \& Holm, 1982). It thus appears that long-term guar-gum treatment in subjects with reduced glucose tolerance can further reduce the postprandial blood glucose levels. However, in subjects with normal blood glucose profiles following a meal, long-term treatment with guar gum does not appear to produce any further effect above and beyond that achieved acutely, i.e. a modest reduction in postprandial blood glucose levels.

Long-term treatment with the granulated guar gum was also associated with a significant reduction in serum cholesterol but unchanged $\alpha$-cholesterol and triglyceride levels. These findings taken together show that guar-gum treatment leads to a reduction in LDL-cholesterol without significantly influencing the HDL-cholesterol. Similar findings have previously been reported in patients with diabetes and hyperlipidaemia and in healthy volunteers (Jenkins et al. 1979, 1980; Smith \& Holm, 1982). Thus, guar gum seems to be an effective agent for lowering cholesterol levels even in the absence of any change in body-weight.

The obese subjects in the present study were asked to maintain their normal dietary habits throughout the study. In spite of this a significant reduction in body-weight was found, suggesting a reduced energy intake. Previous studies have usually reported that guar-gum treatment did not significantly influence body-weight, although this has been found in some investigations (Jenkins et al. 1980; Tuomilehto et al. 1980).

In order to elucidate further the effect of guar gum on appetite regulation in obesity a second experiment was carried out. Since no placebo with the physico-chemical characteristics of guar gum was known, the study was carried out with wheat bran as a reference material. It cannot be excluded that bran has an effect on appetite regulation by itself, but it was not possible to evaluate this in the present work.

The results of the present study clearly demonstrate that guar gum taken in conjunction with meals leads to a reduction in hunger as compared with wheat bran taken in the same way. This effect seems to be long-lasting and could still be demonstrated after a 10 -week period.

It seems reasonable to assume that the reduced hunger and increased satiety reported here is an important reason for the weight reduction found in the obese subjects. The reduction in hunger caused by consumption of guar gum is probably due to its effect on the gastric emptying time. Previous studies, as well as preliminary results with the granulated guar gum (M. Krotkiewski, unpublished results), have shown a slower emptying time when guar gum is taken with the meal, either alone (Wilmshurst \& Crawley, 1980) or in combination with pectin (Holt et al. 1979). The reason for this is probably an increased viscosity of the gastric contents produced by the hydrophilic character of guar gum. In agreement with this, it was recently reported (Wilmshurst \& Crawley, 1980) that guar gum added to a meal increased satiety and that a significant correlation existed between the mean gastric emptying time and the feeling of satiety.

In summary, the present results document some effects of granulated guar gum on carbohydrate and lipid metabolism in obese individuals. These effects would appear of particular importance in obesity considering the known associations with diabetes and lipid disorders. In addition, guar gum reduces hunger when taken with meals and may thus be an important adjunct to other treatments of obesity.

\section{REFERENCES}

Borner, K. \& Klose, E. (1977). Journal of Clinical Chemistry 15, 121-130.

Forbes, G. B., Gallup, J. \& Hurch, J. B. (1961). Science 133, 101-102.

Holt, S., Heading, R. C., Carter, D. C., Prescott, L. F. \& Tothill, P. (1979). Lancet ii. 636-639.

Jenkins, D. J. A., Goff, D. V., Leeds, A. R., Alberti, K. G. M., Wolver, T. M. S., Gasull, M. A. \& Hockaday,

T. D. R. (1976). Lancet ii, 172-174. 
Jenkins, D. J. A., Leeds, A. R., Gassull, M. A., Cochet, B. \& Alberti, K. G. M. M. (1977). Annals of Internal Medicine 86, 20-23.

Jenkins, D. J. A., Leeds, A. R., Slavin, B., Mann, J. \& Jepson, E. M. (1979). American Journal of Clinical Nutrition 32, 16-18.

Jenkins, D. J. A., Reynolds, R., Slavin, B., Leeds, A. R., Jenkins, A. L. \& Jepson, E. M. (1980). American Journal of Clinical Nutrition 33, 575-581.

Jenkins, D. J. A., Wolever, T. M. S., Leeds, A. R., Gassull, M. A., Haisman, P., Dilawari, J., Goff, D., Metz, G. L. \& Alberti, K. G. M. M. (1978). British Medical Journal i, 1392-1394.

Metropolitan Life Insurance Co. (1959). Statistical Bulletin 40, 1-4.

Morgan, L. M., Goulder, T. J., Triolakis, D., Marks, V. \& Alberti, K. G. M. M. (1979). Diabetologia 17, 85-89.

Sköldborn, H. \& Arvidsson, M. (1972). Proceedings of the Sixth International Nordic Meeting on Clinical Physics. Acta Radiologica 313, Suppl., 233.

Smith, U. \& Holm, G. (1982). Atherosclerosis 45, 1-10.

Tuomilehto, J., Voutilainen, E., Huutunen, J., Vinni, S. \& Homan, K. (1980). Acta Medica Scandinavica 208, 45-48.

Wahlefeld, A. W. (1974). In Methods of Enzymatic Analysis, p. 1831 [H. V. Bergmeyer, editor]. New York: Academic Press.

Wiklund, O., Fager, G., Craig, I. H., Wilhelmsson, C.-E., Vedin, A., Olofsson, S.-O., Bondjers, G. \& Wilhelmsen, L. (1980). Scandinavian Journal of Clinical and Laboratory Investigations 40, 239-247.

Wilmshurst, P. \& Crawley, J. C. W. (1980). British Journal of Nutrition 44, 1-6. 